\title{
Four propellers submarine drone modelling in a real environment
}

\author{
Mohamed Moustanir'1, Karim Benkirane ${ }^{2}$, Adil Sayouti ${ }^{3}$, Hicham Medromi ${ }^{4}$ \\ ${ }^{1,3,4}$ Department of Electrical Engineering, Hassan II University, National High School of Electricity and Mechanics, \\ Casablanca, Morocco \\ ${ }^{2,3}$ Royal Naval School, Casablanca, Morocco
}

\begin{tabular}{l} 
Article Info \\
\hline Article history: \\
Received Dec 20, 2020 \\
Revised Sep 24, 2021 \\
Accepted Oct 1, 2021 \\
\hline
\end{tabular}

\section{Keywords:}

Drive architecture

Modelling

ROV

Submarine drone

Thrusters

\begin{abstract}
In order to reduce the hydrodynamic dampers and mechanical elements as rudders, we have in our previous publications proposed our architectural solution of an ROV with only four thrusters without rudders or diving bars. In the results we have justified the choice of the arrangement of the thrusters. Also, we have started the kinematic and dynamic studies of the marine robot and we have especially demonstrated by using the mathematical model under MATLAB in the last publication, that this remotely operated vehicle (ROV) can move in a perfect environment without gravity or hydrodynamic dampers. In this article, we will study the behavior of this marine vehicle in a real environment with gravity and hydrodynamic dampers and we will view if this architectural solution can really allow the ROV to move and execute the given directional instructions.
\end{abstract}

This is an open access article under the CC BY-SA license.

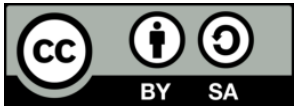

Corresponding Author:

Mohamed Moustanir

Department of Electrical Engineering

Hassan II University

National High School of Electricity and Mechanics "ENSEM", Casablanca, Morocco

Email: mohamed.moustanir@ensem.ac.ma

\section{INTRODUCTION}

Remotely operated vehicles (ROVs), like all underwater vehicles [1], have grown in importance over the years. Their deployment is becoming more and more omnipresent and often unavoidable [2], because the underwater environment is a hostile and formidable environment and makes this type of mission difficult and expensive. Marine robots provide the more than perfect solution for missions [3], involving civil and military fields such as maritime cartography, oceanography, underwater research or seabed surveillance.

Several models and technologies of these machines were appeared, at their beginning, they were remote controlled with the humane presence in the chain of decision, after they created autonomous variants AUVs (autonomous underwater vehicles) which filled the limitations of ROVs. All these submersibles have been able to assert their importance and consolidate their position as an indispensable tool to avoid endangering humans and to reduce operating costs by automating some repetitive or reduced-decision tasks in relation to the fields aforementioned [4].

The propellant solution for torpedo-shaped, used in our work, underwater robots is commonly a rear thruster as shown in Figure 1, while the guiding solution is almost generally a combination of rudders and diving bars to ensure the evolution of the submarine in the aquatic environment in the desired directions. An architectural solution, as shown Figure 2, consists in using four orientable propellers with a pair at the front and another pair at the rear, but this solution supposes the installation of additional mechanisms to handle the 
thrusters and in addition, it also has a Maneuvering thruster in bow. But is it possible to improve or optimize this architectural solution and offer a more advantageous alternative for shifting?

The architectural solution proposed in this study consists of a torpedo-shaped marine robot, but with four propeller thrusters at the rear, which will simultaneously provide propulsion and direction guidance. The principle of this solution is partly inspired by aerial drones with four vertical rotors and at the same time also used as an emergency man oeuvre for surface navigation in the event of rudder failure, but only for ships with more than one propeller. So, to keep a given direction, it is necessary to maintain the same thruster speed and for changing direction it will only be necessary to unbalance the distribution of the thrust of the thrusters in favor of one direction or the other.

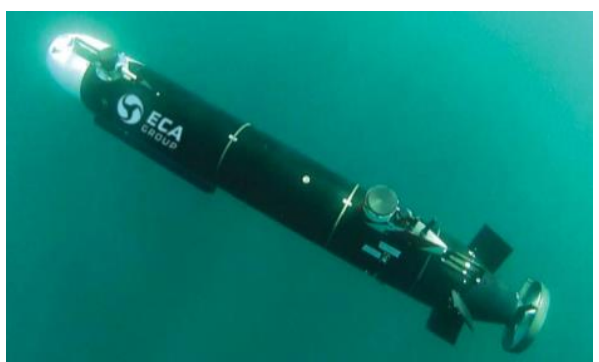

Figure 1. Submarine robot with rear propeller, rudders, and diving bars (ECA group)

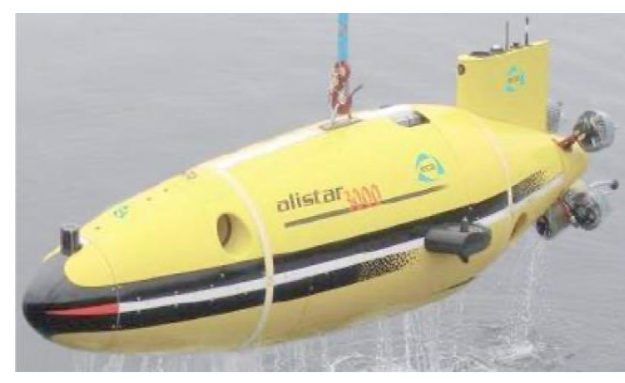

Figure 2. Alistar robot with four rear propellers (ECA group)

\section{RESEARCH METHOD}

\subsection{Presentation}

The general concept of this vehicle was presented in the article [6] which precedes this study, it describes in addition to the shape of this vehicle, the advantages and disadvantages of this architectural solution. In the same way, we were able to demonstrate in our last publication [7] that in a real aquatic environment, this ROV as Figure 3 can move and execute the orders received. Thus, as demonstrated, the most common engine thrust combinations have been tested and the resulting curves have been presented.

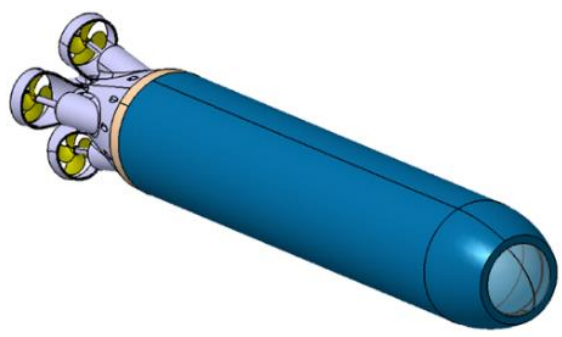

Figure 3. Proposed model in perspective

Two approaches are possible to have a dynamic model [8]: one starting from the conservation of equations of the kinetic and potential energies of Lagrange-Euler [9], [10] and the other developing the fundamental equation of mechanics named method of Newton-Euler [11]. It's this last that was used in this researched works. The modeling of the ROV includes the kinematic part that will focus on the movement and geometric relationships of the submarine. While the dynamic part will deal with the forces and torques acting on this machine.

\subsection{Kinematic model}

The global position vector [12], [13]:

$$
\begin{aligned}
& \eta=[x, y, z, \varphi, \theta, \psi]^{T} \\
& \eta=\left(\eta_{1}, \eta_{2}\right)^{T} \quad \eta_{1}=(x, y, z)^{T} \quad \eta_{2}=(\varphi, \theta, \psi)^{T}
\end{aligned}
$$


In the same way we can combine the speeds:

$$
v=\left(v_{1}, v_{2}\right)^{T} \quad v_{1}=(u, v, w)^{T} \quad v_{2}=(p, q, r)^{T}
$$

In coordinate system $\mathrm{R}_{0}$ :

$$
\dot{\eta}_{1}=J_{C_{1}}\left(\eta_{2}\right) v_{1} \quad \dot{\eta}_{2}=J_{C_{2}}\left(\eta_{2}\right) v_{2}
$$

with:

$$
\begin{aligned}
& J_{C_{1}}\left(\eta_{2}\right)=\left(\begin{array}{ccc}
c \psi c \theta & -s \psi c \varphi+c \psi s \theta s \varphi & s \psi s \varphi+c \psi s \theta c \varphi \\
s \psi c \theta & c \psi c \varphi+s \psi s \theta s \varphi & -c \psi s \varphi+s \psi s \theta c \varphi \\
-s \theta & c \theta s \varphi & c \theta c \varphi
\end{array}\right) \\
& J_{C_{2}}\left(\eta_{2}\right)=\left[\begin{array}{ccc}
1 & \sin \varphi \tan \theta & \cos \varphi \tan \theta \\
0 & \cos \varphi & -\sin \varphi \\
0 & \sin \varphi / \cos \theta & \cos \varphi / \cos \theta
\end{array}\right]
\end{aligned}
$$

The Roll, Pitch and Yaw $(\varphi, \theta$ and $\psi)$ designates the Euler's angles (Figure 4), which in robotics correspond to the system commonly called R.T.L:

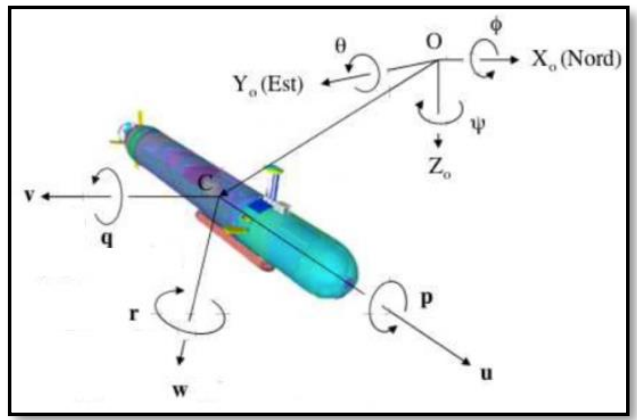

Figure 4. Representation of Euler angles [14]

\subsection{Dynamic model}

In general, the forces generated by the rotation of the earth on the vehicle can be neglected compared to the hydrodynamic forces, if we consider that the $\mathrm{R}_{0}$ coordinate system is a Galilean coordinate system fixed to the surface of the Earth. The dynamic simulation will be inspired by the work of Fossen [9]. In this reference, the fundamental principle of the dynamics applied to the mobile gives [15]:

$$
M \dot{v}=f_{c}+f_{g}+f_{h}+\tau+w
$$

Where the vectors:

- $\dot{v}=[\dot{u}, \dot{v}, \dot{w}, \dot{p}, \dot{q}, \dot{r}]^{T}$ : vehicle accelerations

- $f_{c}$ : forces and torques of drive inertia and Coriolis

- $f_{g}$ : forces and torques induced by weight and Archimedes' thrust

- $f_{h}$ : hydrodynamic forces and torques

- $\tau$ : forces and torques produced by actuators

- $w$ : external disturbances (waves ...)

\subsubsection{Inertia and coriolis}

Any moving body moving in a fluid whose densities are close, causes a displacement of a certain quantity of this fluid during its movement. Thus, from the physical point of view, Newton's second law is no longer applicable in his classical form, because air was admitted as a vacuum and the mass of the displaced air is negligible compared to the mass of the solid in motion, from where it is necessary to take into account 
this displaced mass of fluid [16]. Consequently, the balance of the forces due to the inertia and to this added mass of water must be written in the form:

$$
\sum f_{i}=\left(M_{r}+M_{a}\right) \cdot \gamma_{r}
$$

As a result, relation (8) which concerns the mass matrix, is seen to be developed. The inertia matrix of the fluid is positive and symmetrical. The underwater vehicle has two planes of symmetry, one with respect to the plane (xz) and another with respect to the plane (xy) and since it has a torpedo shape, so we can simplify the symmetries and the matrix can take the following form [17]:

$$
M_{a}=-\left[\begin{array}{cccccc}
X_{\dot{u}} & 0 & 0 & 0 & 0 & 0 \\
0 & Y_{\dot{v}} & 0 & 0 & 0 & 0 \\
0 & 0 & Z_{\dot{w}} & 0 & 0 & 0 \\
0 & 0 & 0 & K_{\dot{p}} & 0 & 0 \\
0 & 0 & 0 & 0 & M_{\dot{q}} & 0 \\
0 & 0 & 0 & 0 & 0 & N_{\dot{r}}
\end{array}\right]
$$

For our ROV:

$$
\begin{aligned}
& X_{\dot{u}}=0,1 m \\
& Y_{\dot{v}}=\rho \pi a^{2} L \\
& Z_{\dot{w}}=\rho \pi a^{2} L \\
& K_{\dot{p}}=0 \\
& M_{\dot{q}}=\frac{\rho \pi a^{2} L^{3}}{12}+\frac{m a^{3}}{15 L} \\
& 0 \\
& N_{\dot{r}}=\frac{\rho \pi a^{2} L^{3}}{12}+\frac{m a^{3}}{15 L}
\end{aligned}
$$

Likewise, for the matrix of inertia and as the center of the reference, Rv coincides with the center of gravity and taking into account the symmetries we can simplify is being as [17]:

$$
M_{v}=-\left[\begin{array}{cccccc}
m & 0 & 0 & 0 & 0 & 0 \\
0 & m & 0 & 0 & 0 & 0 \\
0 & 0 & m & 0 & 0 & 0 \\
0 & 0 & 0 & I_{x x} & 0 & 0 \\
0 & 0 & 0 & 0 & I_{y y} & 0 \\
0 & 0 & 0 & 0 & 0 & I_{z z}
\end{array}\right]
$$

\subsubsection{Weight and Archimedes' thrust}

The forces that the submarine undergoes in water are Archimedes' push and that of its own weight and are written:

$$
\begin{aligned}
& W=m \cdot g \\
& B=\rho \cdot \nabla \cdot g
\end{aligned}
$$

with:

- $m$ : vehicle mass

- $g$ : earth acceleration

- $\rho:$ water density

- $\nabla$ : displaced water volume.

Thus, the vector of hydrostatic forces can be written as [16]: 


$$
f_{g}=\left[\begin{array}{c}
-(W-B) \sin \theta \\
(W-B) \cos \theta \sin \varphi \\
(W-B) \cos \theta \cos \varphi \\
\left(y_{g} W-y_{b} B\right) \cos \theta \cos \varphi-\left(z_{g} W-z_{b} B\right) \cos \theta \sin \varphi \\
-\left(x_{g} W-x_{b} B\right) \cos \theta \cos \varphi-\left(z_{g} W-z_{b} B\right) \sin \theta \\
\left(x_{g} W-x_{b} B\right) \cos \theta \cos \varphi-\left(y_{g} W-y_{b} B\right) \sin \theta
\end{array}\right]
$$

with:

- B: Archimedes' thrust (Buoyancy) in N

- W: Weight in $\mathrm{N}$

- $\left(x_{g}, x_{g}, x_{g}\right)$ : the position of the center of gravity in $\mathrm{Rv}$

- $\left(x_{b}, x_{b}, x_{b}\right)$ : the position of the center of gravity in $\mathrm{Rv}$

If we consider that the center of thrust and the center of the reference $\mathrm{Rv}$, are placed on the same axis $(\mathrm{Oz})$, then, the center of gravity must be below the center of thrust for the submarine to keep its position of initial equilibrium under the effect of the righting moment (Figure 5).

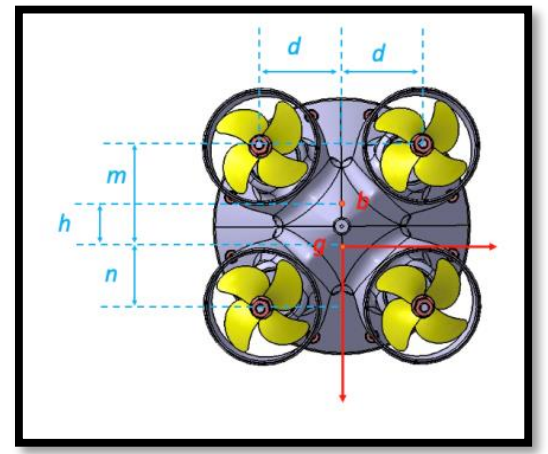

Figure 5. "x" configuration in rear view

From where $x_{b}=0, y_{b}=0$ and $z_{b}=-h$, and that the mass of the submarine is distributed symmetrically with respect to the three planes $(x z),(x y)$ and $(y z)$, we know that in $\mathrm{R}_{\mathrm{V}}: x_{g}=0, y_{g}=$ 0 et $z_{g}=0$, the same at equilibrium $\mathrm{W}=\mathrm{B}$ :

$$
f_{g}=\left[\begin{array}{c}
0 \\
0 \\
0 \\
W h \cos \theta \sin \varphi \\
W h \sin \theta \\
0
\end{array}\right]
$$

\subsubsection{Hydrodynamic forces and torques}

There are other types of hydrodynamic dampers and they affect primarily surface ships rather than underwater vehicles. Thus, it becomes difficult to separate all dampers closely, it is then necessary to express in a global way all these hydrodynamic damping forces in a single term which depends on the speed [18]:

$$
f_{h}=-D(v) \cdot v=-\left(D_{l}+D_{n l}(v)\right) \cdot v
$$

- $D_{l}$ : linear damping.

- $D_{n l}$ : non-linear damping. with: 


$$
\begin{aligned}
D_{l} & =\left[\begin{array}{cccccc}
D_{u} & 0 & 0 & 0 & 0 & 0 \\
0 & D_{v} & 0 & 0 & 0 & 0 \\
0 & 0 & D_{w} & 0 & 0 & 0 \\
0 & 0 & 0 & D_{p} & 0 & 0 \\
0 & 0 & 0 & 0 & D_{q} & 0 \\
0 & 0 & 0 & 0 & 0 & D_{r}
\end{array}\right] \\
D_{n l} & =\left[\begin{array}{cccccc}
D_{n u}|u| & 0 & 0 & 0 & 0 & 0 \\
0 & D_{n v}|v| & 0 & 0 & 0 & 0 \\
0 & 0 & D_{n w}|w| & 0 & 0 & 0 \\
0 & 0 & 0 & D_{n p}|p| & 0 & 0 \\
0 & 0 & 0 & 0 & D_{n q}|q| & 0 \\
0 & 0 & 0 & 0 & 0 & D_{n r}|r|
\end{array}\right]
\end{aligned}
$$

Where the coefficients in $« \|$ " are the absolute values of the linear and absolute speeds.

The submarine will be assumed like a cylindrical shape of length L, with two hemispheres at the end of radius a. As the submarine does not have a drive, rudder or rudders therefore:

$$
\begin{array}{ll}
D_{u}=\frac{X}{u}=\rho \pi\left(2 a L+\pi a^{2}\right) & D_{n u}|u|=\frac{1}{2} \rho \pi a^{2} \\
D_{v}=\frac{Y}{v}=\rho \pi^{2} a^{2} & D_{n v}|v|=\rho a L \\
D_{w}=0 & D_{n w}|w|=\rho a L \\
D_{p}=0 & D_{n p}|p|=\frac{1}{32} \rho a L^{4} \\
D_{q}=0 & D_{n q}|q|=\frac{1}{32} \rho a L^{4} \\
D_{r}=0 & D_{n r}|r|=0
\end{array}
$$

If we consider:

$$
a=0.152 \mathrm{~m} \quad L=1,2 \mathrm{~m} \quad \rho=1020 \mathrm{~kg} / \mathrm{m}^{3}
$$

So, after calculation, the hydrodynamic coefficients specific to this ROV will be grouped in the Table 1 and we will become:

Table 1. ROVs hydrodynamic coefficients [S.I]

\begin{tabular}{cccc}
\hline$D_{u}$ & 1401.56 & $D_{n u}|u|$ & 37.01 \\
\hline$D_{v}$ & 232.58 & $D_{n v}|v|$ & 186.04 \\
$D_{w}$ & 0 & $D_{n w}|w|$ & 186.04 \\
$D_{p}$ & 0 & $D_{n p}|p|$ & 10.04 \\
$D_{q}$ & 0 & $D_{n q}|q|$ & 10.04 \\
$D_{r}$ & 0 & $D_{n r}|r|$ & 0 \\
\hline
\end{tabular}

\subsubsection{External disturbances}

External disturbances affecting a marine vehicle are the wind (only for surface vehicles), waves (for surface or subsurface vehicles), and current, but the most important is the umbilical [17]. But in the presence of the embilical, the dynamic study should be more detailed [19]. Since it has no cable for our ROV and if we consider that the submarine will evolve in calm waters without the presence of waves or currents, these disturbances can be neglected. 


\subsubsection{Actuator effects}

They designate generally any power source which can generate a thrust exerted on the vehicle, in our case, they are four electric motors of brushless type mounted in configuration " $x$ ". Modeling the thrust of a thruster is relatively delicate because it depends on several parameters and is further complicated by the coupling of several thruster. Because in our case, the thrusts of the four thrusters are parallel to the axis Ox. seen from the rear, taking into account the configuration of the thrusters (Figure 5). The vector of forces and torques applied to the vehicle by the actuators is generally defined as [17]:

$$
\tau=\left(f_{x}, f_{y}, f_{z}, \Gamma_{x}, \Gamma_{y}, \Gamma_{z}\right)^{T}
$$

In our case, since we have four thrusters directed along the Ox axis, then:

$$
\tau=\left(f_{x}, 0,0,0, \Gamma_{y}, \Gamma_{z}\right)^{T}
$$

Therefore, the matrix of actuators:

$$
\tau=T . f_{i}
$$

Who becomes:

$$
\tau=\left[\begin{array}{cccc}
1 & 1 & 1 & 1 \\
0 & 0 & 0 & 0 \\
0 & 0 & 0 & 0 \\
0 & 0 & 0 & 0 \\
-d-h / 2 & -d-h / 2 & +d-h / 2 & +d-h / 2 \\
+d & -d & -d & +d
\end{array}\right] \cdot\left[\begin{array}{c}
f_{1} \\
f_{2} \\
f_{3} \\
f_{4}
\end{array}\right]
$$

\subsection{Simulation}

\subsubsection{Numerical resolution}

The MATLAB language will be used for the simulation of the movement of this ROV, for that we will implement the program in this language. Similarly, and depending on the engine controls and also the response of the vehicle, the described trajectory will be visualized on a $3 \mathrm{D}$ interface. The parameters specific to the ROV of this study, as well as the equations which result from the kinematic, and dynamic studies will be integrated into the global program to visualize the various phases of the vehicle simulation. The ROV equation of state system can be defined as:

$$
\left\{\begin{array}{c}
\dot{\eta}(t)=J_{C}(\eta) \cdot v(t) \\
\dot{v}(t)=M^{-1}\left(T \cdot f_{i}-W(\eta)-D(v) \cdot v(t)\right)
\end{array}\right.
$$

with:

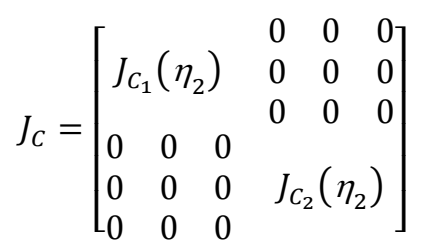

and:

$$
M=-\left[\begin{array}{cccccc}
X_{\dot{u}}+m & 0 & 0 & 0 & 0 & 0 \\
0 & Y_{\dot{v}}+m & 0 & 0 & 0 & 0 \\
0 & 0 & Z_{\dot{w}}+m & 0 & 0 & 0 \\
0 & 0 & 0 & K_{\dot{p}}+I_{x x} & 0 & 0 \\
0 & 0 & 0 & 0 & M_{\dot{q}}+I_{y y} & 0 \\
0 & 0 & 0 & 0 & 0 & N_{\dot{r}}+I_{z z}
\end{array}\right]
$$

Some numerical data specific to the ROV: 


$$
\begin{aligned}
& J_{\Delta y}=J_{\Delta z}=1 / 2 \mathrm{~m} \cdot \mathrm{r}^{2}=3,504 \mathrm{Kg} \cdot \mathrm{m}^{2} \\
& J_{\Delta x}=0,143 \mathrm{Kg} \cdot \mathrm{m}^{2} \\
& r_{1}=60 \mathrm{~mm} \\
& r_{2}=60 \mathrm{~mm} \\
& l=1,2 \mathrm{~m} \\
& m=28,6 \mathrm{Kg} \\
& d=90 \mathrm{~mm} \\
& h=15 \mathrm{~mm}
\end{aligned}
$$

In the following part, we will use the numerical resolution of the differential equations to visualize the dynamic behavior specific to our submarine robot, of which the input parameters of the system will be only the different power configurations of the thrusters. The (28), which govern the model resulting from the kinematic and dynamic studies of the ROV, can be solved by several methods of numerical resolution of differential equations, but in this chapter, we have opted for the 4th order runge-kutta (RK) method for its remarkable precision in solving this type of equation [20].

\subsubsection{Implementation}

This method is an efficient and reliable method for solving differential equations, and also can be used in a general way usable at any order of differential equations. To do this, it suffices to convert the order ' $n$ ' of the equation to be solved to the number ' $n$ ' of equations to be solved. Nevertheless, it requires a meticulous sequencing for its application, because it involves four times more calculation than the Euler method for example. The sequence to apply this method for solving a 1st order differential equation with the 4th order Runge-Kutta method, can be written as [21]:

$$
\begin{aligned}
& y_{(t=0)}=y_{0} \\
& K_{1}=f\left(t_{0}, y_{0}\right) \cdot d t \\
& K_{2}=f\left(t_{0}+\frac{d t}{2}, y_{0}+\frac{K_{1}}{2}\right) \cdot d t \\
& K_{3}=f\left(t_{0}+\frac{d t}{2}, y_{0}+\frac{K_{2}}{2}\right) \cdot d t \\
& K_{4}=f\left(t_{0}+d t, y_{0}+K_{3}\right) \cdot d t \\
& y_{1}=y_{0}+\frac{1}{6} \cdot\left(K_{1}+2 K_{2}+2 K_{3}+K_{4}\right)
\end{aligned}
$$

The diagram of this 4th order method above will be applied in our case for the solution of the equations. But we must first decompose our equation which is at the 2 nd degree in 2 equations of the 1 st degree. the coefficients of RK will be calculated simultaneously and alternately along the resolution process to finally find the desired values as:

$$
\begin{array}{ll}
y_{(t=0)}=y_{0} & h_{(t=0)}=h_{0} \\
K_{1}=f\left(y_{0}\right) \cdot d t & J_{1}=g\left(h_{0}\right) \cdot d t \\
K_{2}=f\left(y_{0}+\frac{J_{1}}{2}\right) \cdot d t & J_{2}=g\left(h_{0}+\frac{K_{1}}{2}\right) \cdot d t \\
K_{3}=f\left(y_{0}+\frac{J_{2}}{2}\right) \cdot d t & J_{3}=g\left(h_{0}+\frac{K_{2}}{2}\right) \cdot d t \\
K_{4}=f\left(y_{0}+J_{3}\right) \cdot d t & J_{4}=g\left(h_{0}+K_{3}\right) \cdot d t \\
y_{1}=y_{0}+\frac{1}{6} \cdot\left(K_{1}+2 K_{2}+2 K_{3}+K_{4}\right) & h_{1}=h_{0}+\frac{1}{6} \cdot\left(J_{1}+2 J_{2}+2 J_{3}+J_{4}\right)
\end{array}
$$

Note: All these coefficients (vectors and matrices) are order 6.

This algorithm will be implemented in MATLAB in the form of a program with the values of the thrusts as inputs. The most common combinations of thrusters will be tested to determine the reaction of the ROV in each case. 


\section{RESULTS AND DISCUSSION}

The system entries indicating the values of the thrusts developed by the thrusters will be given in the order $\left[\mathrm{F}_{1}\right]-\left[\mathrm{F}_{4}\right]$. To have the balance of the thrusts, normally they must have the same values. However, as the center of gravity of the vehicle is not on the axis $(\mathrm{Ox})$, we will have an equilibrium coefficient of horizontal forces. So, this coefficient will indicate the proportionality between the horizontal thrusts and he is defined as:

$$
p=\left(1+\frac{h}{n}\right) \cdot \frac{F_{2}}{F_{3}}
$$

We can see that if $h=0$ we have a proportionality of $p=1$ when $F_{3}=F_{2}$. For example: $F_{3}=$ 22 and $F_{2}=14$. To give a comparative idea between the study in an ideal and real environment, we will try to keep the same configuration and proportionality schemes previously entered.

- Thrusters have proportionally the same thrust $\{18 ; 18 ; 28 ; 28\}$

In this case (Figure 6) the robot keeps a straight path. Only the x position changes, the other five parameters remain zero. Its speed along the Ox axis increases initially, then stabilizes throughout the remainder of the simulation.

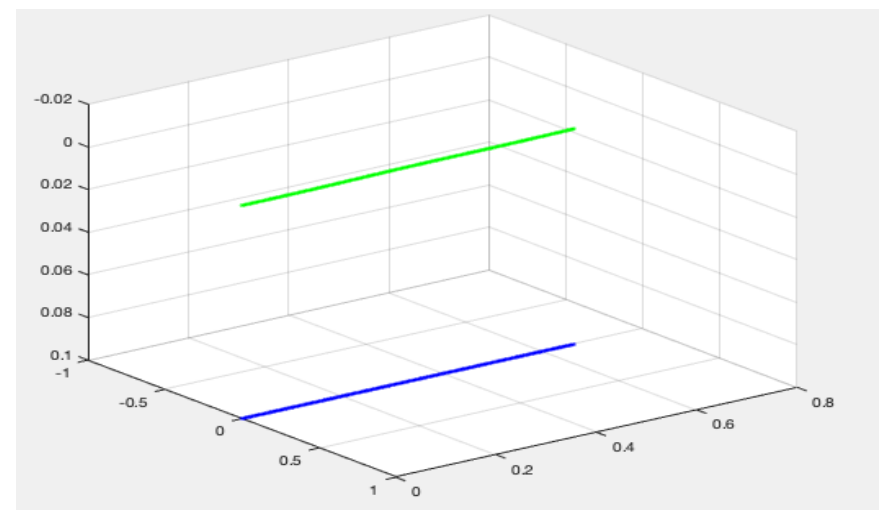

Figure 6. Thrusters have proportionally the same thrust

- Vertical thrusters have proportionally the same thrust $\{15 ; 10 ; 23 ; 28\}$

With this configuration (Figure 7), the ROV describes an arc in a plane parallel to the plane $\mathrm{z}=0$ at constant $\mathrm{z}$, the radius depends on the power of the thrusters and only the angle theta varies.

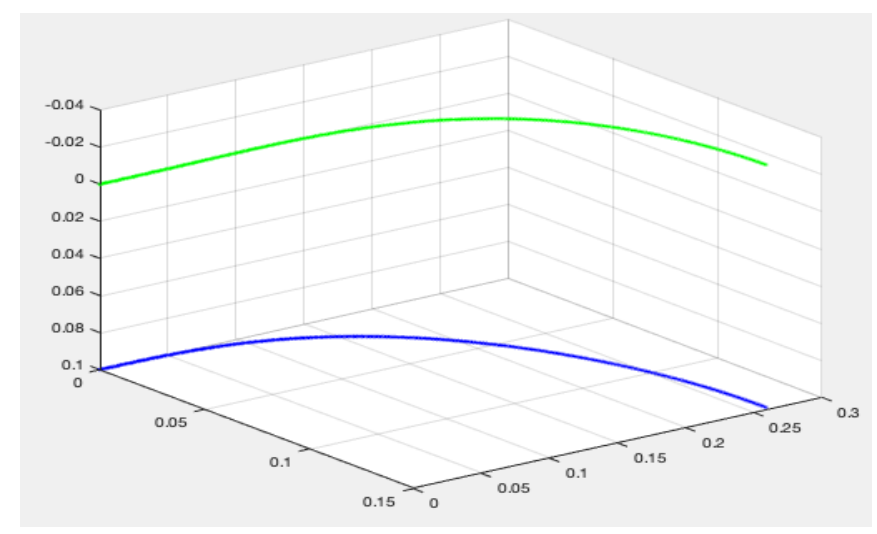

Figure 7. Vertical thrusters have proportionally the same thrust 
- $\quad$ One of the four thrusters is proportionally different to the others $\{18 ; 18 ; 28 ; 0\}$

In this combination (Figure 8), the vehicle will describe a spiral whose radius and pitch will depends on the speed of the ROV and the deference of the power of this thruster with the other thrusters.

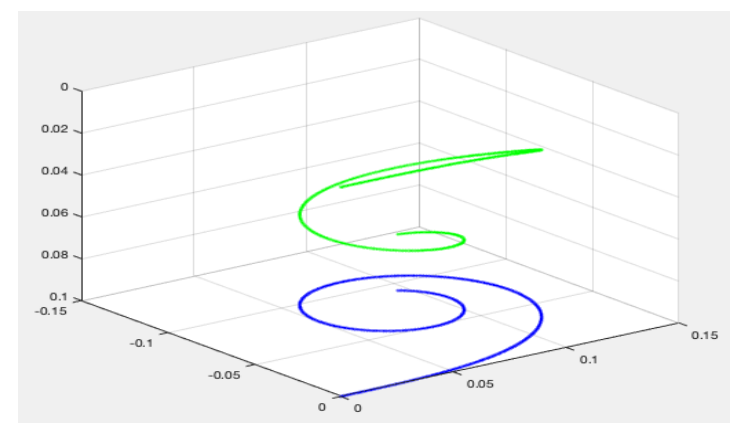

Figure 8. One of the four thrusters is proportionally different to the others

To confirm our results in practice, we started the realization phase in our Laboratory in the ENSEM Hassan II University. The production part is almost finished, prototyping and 3D printing are at a very advanced stage. In these figures (Figures 9 to 11), we have seen how the ROV is partly carried out, the assembly and the wiring are in an advanced stage, remain the dry tests, to then begin those in real conditions.

Several methods are used to vary the signal of motor controls such as pulse width modulation (PWM) [21] (accessed May, 2021).

[1] which is an efficient and widely used process including the naval sector, or with a control board dedicated to this task by using an URAT protocol (Universal Asynchronous Receiver/Transmitter) [22], [23]. , doi: 10.11591/ijpeds.v11.i4.pp1701-1710.

However, we will first start with the control through the ESC (Electronic Speed Control) which is compatible with our brushless motors. For the control, we did not opt for an ambilical cable because it will limit the maneuvers of the robot [24], and we chose that the control of this robot will be remote and will be based on an arduino HC12 transceiver module. Its frequency range is between $433 \mathrm{MHz}$ and $473 \mathrm{MHz}$ which is already presented in a study [25], remains its adaptation for these modules for a greater depth.

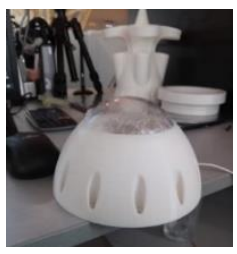

Figure 9. The ROV's bow with the visor

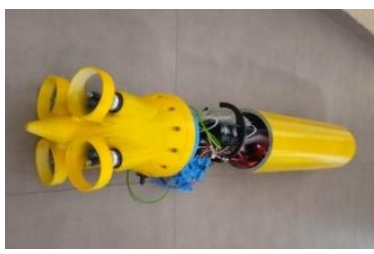

Figure 10. ROV's assembly

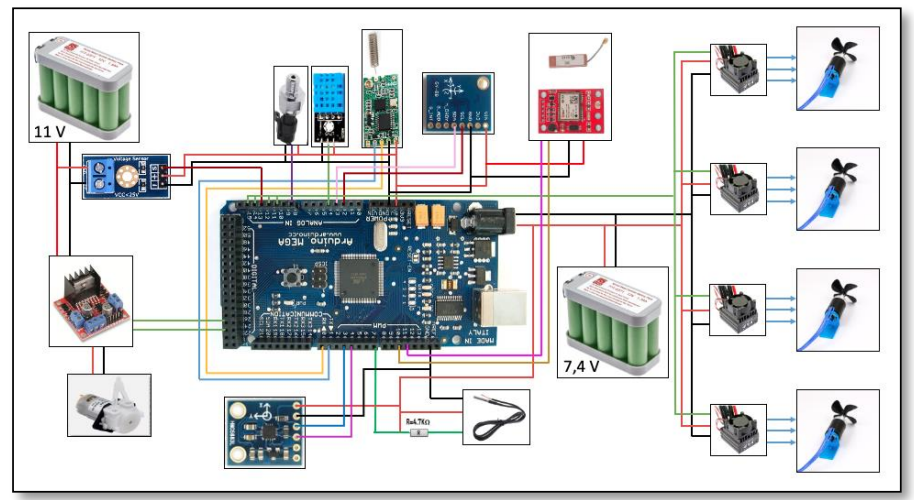

Figure 11. The control and wiring diagrams 


\section{CONCLUSION}

In this paper we were able to show theoretically that this submarine drone with four thrusters can move in a real aquatic environment without having to use rudders or diving bars. The various thrust combinations of the thrusters and the most common ones are tested and we have achieved the expected results. This justifies the choice of our solution. With the practical realization which is in progress, we can after confirm these theoretical obtained results and then proceed to the adjustments and finishes which are essential. In our next studies, we plan to expand the ROV control system with the tests and all the resulting optimizations.

\section{REFERENCES:}

[2] D. Terracciano, L. Bazzarello, A. Caiti, R. Costanzi, and V. Manzari, "Marine robots for underwater surveillance," Current Robotics Reports, vol. 1, pp. 159-167, 2020, doi: 10.1007/s43154-020-00028-z.

[3] R. B. Wynn et al., "Autonomous underwater vehicles (AUVs): Their past, present and future contributions to the advancement of marine geoscience," Marine Geology, vol. 352, pp. 451-468, 2014, doi: 10.1016/j.margeo.2014.03.012.

[4] E. C. Joseph, and G. O. Moses, "Prototype development of tethered underwater robot for underwater vessel anchor release" International Journal of Robotics and Automation (IJRA), vol. 9, no. 3, pp. 196-1210, 2020, doi: 10.11591/ijra.v9i3.pp196-210.

[5] S. A. Mostafa, and A. M. M. Brisha, "Analysis and simulation of 3D trajectory with obstacle avoidance of an autonomous underwater vehicle for optimum performance," International Journal of Computer Science and Network Security (IJCSNS), vol. 12 no. 3, pp. 43-50, March 2012.

[6] M. Moustanir, K. Benkirane, A. Sayouti, and H. Medromi, "Four propellers architecture proposed for the submarine drone," International Research Journal of Engineering and Technology (IRJET), vol. 6, no. 11, pp. 3501-3505, 2019.

[7] M. Moustanir, K. Benkirane, A. Sayouti, and H. Medromi, "Four propellers submarine drone modelling," International Research Journal of Engineering and Technology (IRJET), vol. 6, no. 12, pp. 2084-2090, 2019.

[8] M. Moustanir, K. Benkirane, A. Sayouti, and H. Medromi, "Four propellers underwater drone modelling," in ICCSRE'2020, E3S Web of Conferences, 2021, vol. 229, doi: 10.1051/e3sconf/202122901010.

[9] D. Phuong Nam, N. Hong Quang, T. Phuong Nam, and T. Thi Hai Yen, "Adaptive dynamic programing based optimal control for a robot manipulator," International Journal of Power Electronics and Drive System (IJPEDS), vol. 11, no. 3, pp. 1123-1131, 2020, doi: 10.11591/ijpeds.v11.i3.pp1123-1131.

[10] T. I. Fossen, Guidance and control of ocean vehicles, New York: John Wiley and Sons, 1994.

[11] A. Mallem, N. Slimane, W. Benaziza, "Dynamic Control of Mobile Robot Using RBF Global Fast Sliding mode", International Journal of Robotics and Automation (IJRA), Vol. 7, No. 3, pp. 159 168, September 2018.

[12] W. Li, Z. Zhou, J. Lou, and X. Zhang, "A 3D trajectory tracking algorithm for AUV," Journal of Physics: Conference Series, vol. 1873, 2021 2nd International Workshop on Electronic communication and Artificial Intelligence (IWECAI 2021), 12-14 March 2021, Nanjing, China, doi: 10.1088/1742-6596/1873/1/012055.

[13] Society of Naval Architects and Marine Engineers, Nomenclature for treating the motion of a submerged body through a fluid, New York: Society of Naval Architects and Marine Engineers, 1950.

[14] B. S. Seifallah, "Design of a hybrid coordination algorithm for groups of communicating underwater robots. Application: systematic and detailed optical acquisition of the seabed," (in French), Ph.D. dissertation, Université de Bretagne occidentale - Brest, 2016.

[15] K. J. Kim, Y. H. Choi, and J. B. Par, "Design of path tracking controller for underactuate autonomous underwater vehicle using approach angle concep," Journal of Korean Institute of Intelligent Systems, vol. 22, no. 2, pp. 225231, 2012, doi: 10.5391/JKIIS.2012.22.2.225.

[16] V. Creuze, and B. Jouvencel, "Seabed perception and following for autonomous underwater vehicles," Traitement du Signal, vol. 20, no. 4, pp. 323-336, 2003.

[17] A. Hattab, "Robust modeling and control of a robot," (in French), Ph. Doctoral Thesis, Dept. Electrotechnique, Université des Sciences et de la Technologie d'Oran Mohamed Boudiaf, Alger Oran,2016.

[18] V. Creuze, Marine and submarine robot -Perception, modeling and control, (in French), Techniques de l'Ingénieur, 2014.

[19] L. Jaulin, Mobile robotics, (in French), ISTE Editions Ltd, Great London Britain, 2015.

[20] C. Zhao, P. Thies, J. Lars, and J. Cowles, "ROV launch and recovery from an unmanned autonomous surface vessel -Hydrodynamic modelling and system integration," Ocean Engineering, vol. 232, 15 July 2021, doi: 10.1016/j.oceaneng.2021.109019.

[21] E. Hairer, S.P. Norsett, and G. Wanner, Solving ordinary differential equations I, non stiff problems, Springer Series in Comput. Math, vol. 8, 2nd edition. 1993, doi: 10.1007/978-3-540-78862-1.

[22] J. Geandrot, "Numerical method: Euler's method of Runge-Kutta of order 4 for first or second order equations," (in French), Cours de physique-chimie tous niveau, http://www.physagreg.fr/ (accessed May, 2021).

[23] M. Hasoun, A. El Afia, M. Khafallah, and K. Benkirane, "Experimental implementation a PWM strategy for dual threephase PMSM using 12-sector vector space decompositionapplied on electric ship propulsion," International Journal of Power Electronics and Drive System (IJPEDS), vol. 11, no. 4, pp. 1701-1710, 2020, doi: 10.11591/ijpeds.v11.i4.pp1701-1710. 
[24] Y. M. Ahmed, O. Yaakob, and B. K. Sun, "Design of a new low cost ROV vehicle," Jurnal Teknologi (Sciences \& Engineering), vol. 69, no. 7, pp. 27-32, 2014, doi: 10.11113/jt.v69.3262.

[25] F. Campagnaro, A. Signori, and M. Zorzi, "Wireless remote control for underwater vehicles," Journal of Marine Science and Engineering (JMSE), vol. 8, no. 10, p. 736, Sep. 2002, doi: 10.3390/jmse8100736.

[26] M. I. Sani, S. Siregar, A. P. Kurniawan, and M. A. Irwan, "FIToplankton: Wireless controlled remotely-operated underwater vehicle (ROV) for shallow water exploration," International Journal of Electrical and Computer Engineering (IJECE), vol. 8, no. 5, October 2018, pp. 3325 3332, doi: 10.11591/ijece.v8i5.pp3325-3332.

\section{BIOGRAPHIES OF AUTHORS}
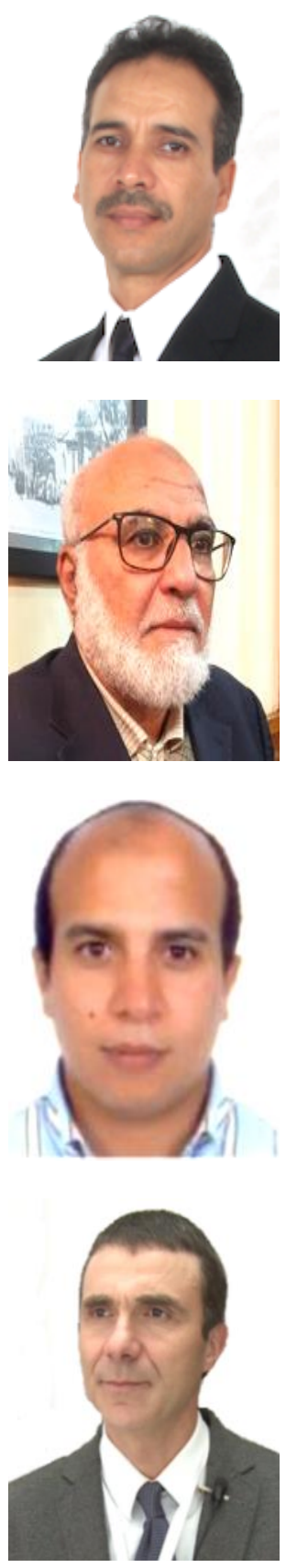

Mohamed Moustanir born in Casablanca in 1971, He received the naval engineering degree in 1995 from Navy Royal School in Casablanca, Morocco, then received his mechanical engineering degree in 2010 at HSU University, Hamburg, Germany. Occupied the function of propulsion engineering chief in various shifs. Currently instructor at the Engineering Department (Mechatronic) in the Royal Navy School and a doctoral student in electrical engineering in ENSEM in Casablanca, His current research interests are Robotic and her applications. Email: mohamed.moustanir@ensem.ac.ma.

Karim Benkirane was born in Morocco in 1960. He received B.Sc., M.Sc. and Doctorate degrees from Mohamed V University Rabat and University of Sciences and Technics at Languedoc (USTL), France, in 1978, 1983 and 1986 respectively, all in Electronics Engineering. In 1987 he joined the Royal Navy School (ERN), Casablanca, Morocco, where he is currently professor tutor in the Department Research. His main research interests the application of powerelectronics converts and motor drives. He has published a lot of research papers in international journals. Email: karbenkirane@yahoo.fr.

Adil Sayouti received the PhD in computer science from the ENSEM, Hassan II in July 2009, Morocco. In 2003, he obtained the Microsoft Certified Systems Engineer. In 2010 he joined the Royal Navy School (ERN), Casablanca, Morocco, where he is currently professor tutor in the Department Research. His main research interests the remote controling. He has published a lot of research papers in international journals. Email: sayouti@gmail.com.

Hicham Medromi received the $\mathrm{PhD}$ in engineering science from the Sophia Antipolis University in 1996, Nice, France. He was director of the National Higher School of electricity and Mechanics, Hassan II University Morocco. He is research director in the Department of Computer Sciences in the Hassan II University, Casablanca. His actual main research interest concerns the control architecture of mobile systems based on multiagents systems. He has a lot of publications and citations in international journals. Email: hmedromi@yahoo.fr. 